\title{
O DESENVOLVIMENTO PROFISSIONAL DE PROFESSORES E O USO DE CASOS DE ENSINO ${ }^{1}$
}

\author{
Isa Mara Colombo Scarlati DOMINGUES ${ }^{2}$
}

RESUMO: O presente texto traz o recorte de uma pesquisa que se situa nas discussões sobre a formação de professores e que investigou a aprendizagem e o desenvolvimento profissional de docentes ao fazerem uso de casos de ensino e de métodos de casos, enquanto ferramenta formativa e investigativa propulsora de processos reflexivos sobre os conhecimentos da docência. O processo de pesquisa e intervenção realizou-se por meio do Portal dos Professores - UFSCar, no curso: "Casos de Ensino e Teorização de Práticas Pedagógicas - professores alfabetizadores", na perspectiva de formação continuada de dezesseis docentes da mesma etapa do Ensino Fundamental I (Anos Iniciais $-1^{\circ}, 2^{\circ}$ e $3^{\circ}$ ano), que atuam como alfabetizadoras. A principal fonte de dados, que orientou a organização do artigo, foi uma das atividades realizadas no Módulo II, destinado à análise de casos de ensino, denominada de vídeo-caso. Para realização deste estudo, de abordagem qualitativa, a pesquisa teve como objetivo analisar os processos reflexivos vividos por professoras alfabetizadoras ao fazerem uso dos casos de ensino, em ambiente virtual, enquanto possibilidade formativa e investigativa. Para tanto, foi utilizado um vídeo-caso que retratasse um evento relacionado ao cotidiano de uma professora alfabetizadora. Os resultados alcançados na pesquisa sinalizam a potencialidade dos casos de ensino na/para a aprendizagem e para o desenvolvimento profissional da docência e apontam que é possível evidenciar pontos que merecem destaques e outros que merecem ser repensados para futuros processos formativos e investigativos utilizando a experiência com vídeo-caso.

PALAVRAS-CHAVE: Casos de ensino. Formação continuada de professores alfabetizadores. Processos reflexivos. Aprendizagem e desenvolvimento profissional da docência.

\section{Introdução}

A reflexão em torno dos processos formativos dos professores é objeto de grande preocupação de pesquisadores da Educação, e dessa forma ganha espaço há algum tempo no cenário educacional. Sensível à preocupação sobre a formação de professores capazes de refletirem sobre suas práticas pedagógicas, busquei caminhos

\footnotetext{
${ }^{1} \mathrm{O}$ presente texto, com algumas adequações e recortes, foi extraído da Tese de Doutorado intitulada "Desenvolvimento Profissional de Professoras Alfabetizadoras em Ambiente Virtual de Aprendizagem: contribuições de Casos de Ensino", defendida em fevereiro de 2013 na Universidade Federal São CarlosUFSCar (São Carlos - SP - Brasil), orientada pela Profa. Dra. Maria da Graça Nicoletti Mizukami.

${ }^{2}$ UFG - Universidade Federal de Goiás. Faculdade de Educação. Jataí - GO - Brasil. $75802-565$ isa.scarlati @gmail.com.
} 
para entender diferentes abordagens ao que se refere aos processos de aprendizagem da docência.

Nessa direção, venho me dedicando, desde o mestrado (2005-2007), ao trabalho com os casos de ensino, narrativas de episódios escolares, que possibilitam aos seus usuários a reflexão a partir da prática docente, à medida que trazem descrições que ilustram e detalham situações da trajetória formativa e profissional, permitindo o estabelecimento de relações entre a teoria e a prática. Dentre as muitas características, os casos de ensino possibilitam a reflexão em qualquer momento do processo de aprendizagem, com diferentes grupos de professores, em diferentes modalidades de ensino, de temáticas variadas, em diferentes formatos dentre outras.

Acrescento que os casos de ensino se destacam como um importante instrumento de interface entre formação e investigação no processo de aprendizagem e desenvolvimento profissional da docência, quando comparados a outros processos formativos.

Para tanto, objetivando fazer uma revisão bibliográfica sobre os processos formativos, destaco alguns teóricos, nacionais e internacionais, que se dedicam aos estudos da formação de professores com diferentes abordagens e que dão direcionamentos para o trabalho com os casos de ensino: Alarcão, Infante e Silva (2000); Domingues Scarlati (2008); Duek (2011); Marcelo Garcia (1999); Merseth (1996); Mizukami (2004); Mizukami et al. (2002); Nono (2001, 2005); Nóvoa (1992); Nóvoa e Finger (2010); Pimenta e Ghedin (2002); Sarmento (2009); Schön (2000); J. Shulman (1992, 2002, 2003); L. Shulman (1986, 1987); Soares (2004); Wassermann (1994a, 1994b) dentre outros.

Nesse contexto, a pesquisa traz como objetivo analisar objetivo analisar os processos reflexivos vividos por professoras alfabetizadoras ao fazerem uso dos casos de ensino, em ambiente virtual, enquanto possibilidade formativa e investigativa.

A partir dessa perspectiva metodológica, a pesquisa foi realizada com os seguintes sujeitos/locus: dezesseis professoras, da mesma etapa do Ensino Fundamental I (Anos Iniciais $-1^{\circ}, 2^{\circ}$ e $3^{\circ}$ ano), que atuam como alfabetizadoras, por meio de um Ambiente Virtual de Aprendizagem (AVA), via Portal do Professores - UFSCar, no curso: "Casos de Ensino e Teorização de Práticas Pedagógicas - professores alfabetizadores" (PORTAL..., 2014).

Para investigar o uso dos casos de ensino, no processo de aprendizagem junto às alfabetizadoras, optei por selecionar os casos que mais se aproximavam da realidade de 
trabalho das professoras, com o intuito de observar a contribuição (ou não) dos casos para o desenvolvimento profissional da docência. Esses casos foram organizados de forma que estimulassem nas alfabetizadoras reflexões sobre a trajetória profissional e as situações escolares enfrentadas ao ensinarem conteúdos de linguagem/Língua Portuguesa.

Assim, nesse artigo apresento uma experiência com um caso de ensino denominado de vídeo-caso, que representa um recorte dos dados extraídos do Módulo II do curso "Casos de Ensino e Teorização de Práticas Pedagógicas - professores alfabetizadores".

A proposta de usar um vídeo-caso foi pensada na tentativa de explorar ainda mais os recursos que o ambiente virtual do Portal dos Professores disponibilizava, nesse caso, a plataforma Moodle. Esse vídeo-caso retrata um episódio escolar disponível no YouTube e expõe uma situação em que duas crianças, Lucas e Jaime, dialogam e discutem suas hipóteses sobre a escrita da palavra "morango" ao fazerem um ditado de palavras com figuras, mediado pela professora.

\section{Casos de ensino e processos reflexivos: "vídeo-caso"}

No decorrer do desenvolvimento do curso, introduziu-se ao planejamento inicial um episódio de ensino, com duração de 03h08min, que denominei de "Vídeo-Caso", disponibilizado no YouTube: "Atividades de escrita com lista de frutas". 
Figura 1 - Vídeo-Caso: Atividades de escrita com lista de frutas.

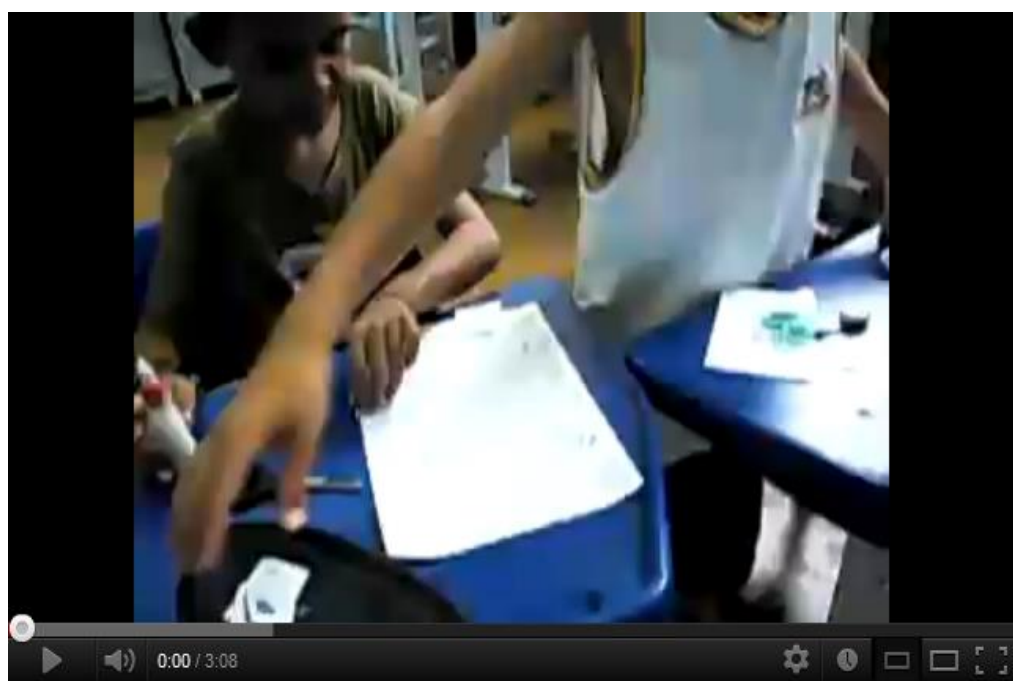

Fonte: ATIVIDADES..., 2014.

O vídeo-caso expõe uma situação em que duas crianças, Lucas e Jaime, fazem um ditado de palavras utilizando gravuras de frutas: morango, tomate, banana, uva e caqui, e de letras do alfabeto, todos disponibilizadas pela professora. Na ocasião, os alunos dialogam e discutem suas hipóteses sobre a escrita da palavra "morango", mediados por algumas intervenções da professora.

As discussões do vídeo-caso, diferentemente dos demais, por suas características de vídeo-gravação e não texto escrito, adotado usualmente, foram realizadas exclusivamente no "Fórum de Vídeo-Caso". Dessa forma não foram propostas questões para serem respondidas, em função das interações acontecerem exclusivamente via fórum. As discussões foram disparadas inicialmente pela formadora e depois pelos próprios cursistas, que sugeriram alguns temas (atividade proposta, metodologia, intervenções etc.) e aconteceram em um único tópico. Dentre as temáticas mais discutidas, algumas abordagens foram muito frequentes: atividade em grupo (duplas); a escrita de lista de palavras; o papel da intervenção do professor nos agrupamentos; os métodos de alfabetização (método fônico). O decorrer dessas discussões revela temáticas que giram em torno do ensino e aprendizagem da leitura e da escrita, com ênfase na adequação ou não do uso de métodos fônicos. Em suma, as discussões do "Fórum de Vídeo-Caso" foram intensas, permitindo abordar alguns conteúdos de linguagem e refletir sobre aspectos da prática da professora. 
Dentre as reflexões desse episódio escolar surgiram também alguns questionamentos: Pode-se pensar em outras formas de agrupamento? Que avaliação pode-se fazer da intervenção da professora? A atividade é adequada para a alfabetização? A professora sinaliza uma prática de alfabetização por meio de qual método? Por essas e outras peculiaridades, esse fórum exigiu intervenções mais pontuais da formadora.

A criação desses espaços de interação permite que todos se comuniquem como iguais, estimulando um processo de aprendizagem sem hierarquização. Com o tempo as alfabetizadoras passaram a se conhecer, perceberem que estavam juntas e com um objetivo comum, sentindo-se valorizadas e reconhecidas pelo que sabem e fazem. Com isso, ficaram mais a vontade para tornar público aquelas reflexões que faziam de formas "solitárias", a ponto de $\mathrm{P} 7^{3}$ postar uma atividade trabalhada com seus alunos, no fórum de vídeo-caso, para que as demais avaliassem o seu trabalho. Diante das contribuições das colegas e da formadora, que a levaram a refletir sobre outras formas de trabalhar a atividade, ela reaplicou a tarefa aos alunos e postou novamente no fórum.

Usa-se Hammernnas, Darling-Hammond e Shulman (2002, p.2) quando afirmo que P7 foi capaz de "voltar atrás, analisar, refletir e aprender com sua experiência dentro de uma comunidade de aprendizes". Acredito também que a discussão estimulada por P7, sobre os métodos de alfabetização, foi um importante desequilibrador nas reflexões sobre a prática. Avalio que o dinamismo das interações, discussões e reflexões, só foi possível pelas características "coletivizadas" e dialógicas dos fóruns.

Conclui-se que a experiência com "Fórum de Vídeo-Caso" foi muito bem avaliada pelas cursistas. Das dezesseis cursistas, treze avaliam como uma experiência "ótima" e três como "boa". Esse fórum foi uma experiência interessante e nova, se comparado à edição anterior do curso de formação com casos de ensino, via Portal dos Professores. Mesmo assim, pode-se dizer que essa atividade foi uma experiência significativa e necessita ser replicada em outros processos formativos e ambientes virtuais para que se possam fazer outras análises sobre seu uso ${ }^{4}$.

\footnotetext{
${ }^{3}$ Para manter a privacidade dos sujeitos, as professoras participantes da pesquisa serão identificadas pelas siglas $\mathrm{P} 1, \mathrm{P} 2$... P16.

${ }^{4}$ Encontra-se no site da INTIME (Integrating New Technologies Into the Methods of Education) algumas experiências com o uso de vídeos em processos formativos. Disponível em: <http://www.intime.uni.edu/vidsearch/Display/Display_All_Videos.asp>. Acesso em: 26 mai. 2014.
} 


\section{"Fórum de vídeo-caso": um ambiente de aprendizagem virtual}

As discussões via fórum tiveram um papel de grande importância no desenvolvimento do curso e foram um instrumento de contato mais próximo entre as cursistas. Isso é ilustrado pelas professoras que destacam a possibilidade das interações com outras professoras alfabetizadoras, que moram em diferentes regiões do país, que vivenciam situações semelhantes, embora atuem em realidades tão diversas. P2 (Q.A.) ${ }^{5}$ assim descreve: “[...] éramos um grupo de professoras alfabetizadoras, com muitas vivências, anseios e dúvidas, e, ao partilhar isso, crescemos juntas”. Para P3 (Q.A.):

[...] cada uma tem uma experiência diferente, cada uma acrescenta uma ideia nova, muitas trocas foram feitas, de atividades, de projetos, de maneiras de agrupamento dos alunos, de prática em sala de aula. Principalmente trocamos dúvidas, medos, aprendemos com a experiência do colega, ajudamos também [...].

$\mathrm{Na}$ opinião de P13 (Q.A.) essas diversidades "demonstraram que apesar das diferenças regionais, há uma sintonia nos desejos pedagógico dos professores com relação à aprendizagem dos alunos, muito mais do que discordâncias".

Essas interações foram intensas e tornaram-se significativas para as alfabetizadoras a ponto de P4 (Q.A.) fazer a seguinte revelação:

Sinto falta de pessoas como a P6, que desabafam o que realmente sentem e são. Aqui, na minha cidade, nenhum professor admite os erros e nem querem refletir sobre a prática, então, se o faço, viro motivo de críticas. Aqui, no curso, me senti acolhida, além de ficar realmente comovida com a humildade de todos [...].

Para P11 (Q.A.), a cursista mais velha e mais experiente do grupo de professoras alfabetizadoras que concluiu o curso,

[...] o contato virtual com as cursistas foi bastante interessante. Interagir com pessoas de diferentes lugares proporcionou uma experiência muito boa. Apesar de atuar como alfabetizadora há 24 anos, aprendi muito com a troca de conhecimentos e, certamente, colocarei em prática muitos dos saberes repassados pelas colegas [...].

${ }^{5}$ Dados extraídos do Questionário de Avaliação (Q.A.). 
O "contato virtual” (Q.A., P11), por suas características, se transforma em um espaço de escuta, fala, discussão e reflexão. Outro aspecto interessante é que as diferenças de idade, de experiência, de local de atuação entre outras, não foram entraves para as aproximações e discussões, ao contrário, foram elementos de reflexão e aprendizagem. Isso pode ser explicado quando Tancredi, Reali e Mizukami (2005, p.33) afirmam que:

As comunidades virtuais são redes eletrônicas de comunicação interativa, organizadas em torno de um interesse ou finalidade comum, abarcando diferentes interesses, valores, concordâncias, e até mesmo discordância e conflitos. A construção das comunidades ocorre por meio dos contatos estabelecidos e se fortalece em função das afinidades de interesse, de conhecimento, de projetos compartilhados.

As cursistas para fazerem suas apreciações sobre o curso também trazem dados importantes sobre o uso de ferramentas da $\mathrm{EaD}$, como quando utilizam, além de um portal virtual, um vídeo-caso. Dentre essas se seleciona duas sobre o ambiente virtual, enquanto espaço de aprendizagem.

Você [P10] resumiu este curso com as melhores palavras que poderíamos usar para definir o quanto aprendemos: "mesmo virtual, foi tão presencial em minha prática pedagógica". Realmente, este curso foi ao mesmo tempo rico em aprendizagens (na troca de experiências) e também foi uma maneira de conseguirmos relatar nossas dúvidas e medos, percebendo que somos PROFESSORES que, mesmo percebendo alguns erros, buscamos refletir e estudar para conseguir uma prática mais efetiva... (F.A., P3) ${ }^{6}$.

A oportunidade que tivemos de nos conhecermos, trocarmos experiências e saberes, foi imensamente rica! O que mais ficou pra mim, foi o acolhimento, mesmo sendo em um ambiente virtual, à distância. Além das reflexões proporcionadas, os apontamentos das colegas me dão a sensação de não estar sozinha. De que esta luta árdua é compensadora, que os erros acontecem e aprendemos continuamente (F.A., P15).

Esses relatos são de grande importância para pensarmos no papel da EaD nos cursos de formação continuada, já que P3 e P15, autoras dos relatos acima, estão entre as professoras que afirmam, no questionário de inscrição, nunca terem participado de um curso de formação na modalidade à distância.

${ }^{6}$ Dados extraídos do Fórum de Avaliação (F.A.). 
O ambiente virtual como espaço de aprendizagem é sinalizado por Moran (2011, p.1) ao dizer que:

[...] a EAD está contribuindo para superar a imagem de individualismo, de um aluno solitário, isolado em um mundo de leitura e atividades distantes do mundo e dos outros. A Internet traz a flexibilidade de acesso, a possibilidade de interação e participação e a combinação da aprendizagem off-line (acesso quando a pessoa quiser) e on-line (de estar junto, de orientar, de tirar dúvidas, de trocar resultados).

Palloff e Pratt (2002) também destacam que o "poder" de uma comunidade de aprendizagem online vai além de um instrutor e de alunos interagindo. É a criação de um espaço, em que todos podem se conectar como iguais em um processo de aprendizagem. Logo eles passam a se conhecer, sentir que estão juntos em alguma coisa e trabalhando com um fim comum.

\section{Considerações}

De modo geral, o trabalho com casos de ensino permitiram que conhecimentos referentes ao ensino e aprendizagem da linguagem fossem explicitados e (re)avaliados pelas alfabetizadoras. As análises apontam que a utilização de casos de ensino e de métodos de casos permitiu que as reflexões se fizessem presentes e que evidenciassem as dúvidas, as certezas e as contradições que orientam e caracterizam a prática profissional das alfabetizadoras.

Posso dizer, pelas discussões, que a experiência com o vídeo-caso foi significativa, pois ajudou as alfabetizadoras tornarem explícitos seus conhecimentos tácitos, a exemplo de P7. Acredita-se que em outras situações o vídeo-caso não deveria ser deixado, como no nosso caso, para o final do curso, momento em que os participantes estão concluindo as atividades. Isso, de certa forma, limitou a participação de todas as alfabetizadoras, que acessaram o fórum, segundo dados extraídos da plataforma moodle, mas muitas vezes não participaram com mensagens.

Finalizo com os estudos de Wassermann (1994b) quando afirma que os filmes são, em certo sentido, outras formas de casos. Há personagens que se identificam com as narrativas, com o considerável teor dramático e com o exame das questões que produzem. Segundo a autora, alguns professores têm selecionado certos filmes como casos e utilizado a metodologia de caso de ensino com sucesso. 
THE PROFISSIONAL DEVELOPMENT OF TEACHING AND THE USE OF TEACHING CASES

ABSTRACT: The present text represents is part of a research that lies in discussions about teacher education and investigated the learning and teachers professional development by making use of teaching cases and case methods, while training tool and investigative processes driving reflective about the knowledge of teaching. The research and intervention process took place through the Teachers Portal - UFSCar in progress: "Casos de Ensino e Teorização de Práticas Pedagógicas - professores alfabetizadores" [Cases of Teaching and Theorizing Pedagogical Practices - literacy teachers], the prospect of continued training of sixteen teachers in the same stage of elementary school (Early Years - 1st, 2nd and 3rd year), which act as literacy. The main source of data, which motivated the organization of the article, was one of the activities in Module II aimed at analyzing teaching cases, called video-case. To accomplishment this study, of qualitative approach, the research aimed to analyze the reflective processes experienced by literacy teachers to make use of teaching cases in the virtual environment, while the possibility formative and investigative. Thus, we used a video if that depicted an event related. The achieved results in the research indicate the potential of teaching cases in / for learning and for professional development of teaching indicated that it is possible to highlight points deserve highlights and others who deserve to be reconsidered for future training and investigative processes using the experience with video case.

KEYWORDS: Case teaching. Continuing education of literacy teachers. Reflective processes. Learning and professional development of teaching. Online training for teachers.

\section{REFERÊNCIAS}

ALARCÃO, I.; INFANTE, M. J.; SILVA, M. S. Descrição e análise interpretativa de episódios de ensino: os casos como estratégia de supervisão reflexiva. In: ALARCÃO, I. (Org.). Formação reflexiva dos professores: estratégias de supervisão. Porto, PT: Editora Porto, 2000. p.151-169.

ATIVIDADES de escrita com lista de frutas. Disponível em: <http://www.youtube.com/watch?v=AKy5XjseKZE\&playnext=1\&list=PL0C1618933F 7A3AB0\&feature=results_main>. Acesso em: 26 mai. 2014.

DOMINGUES SCARLATI, I. M. C. Os Casos de ensino como "potenciais reflexivos" no desenvolvimento profissional dos professores da escola pública.

2007. 157f. Dissertação (Mestrado em Educação) - Instituto de Educação, Universidade Federal de Mato Grosso, Cuiabá, 2008.

DUEK, V. P. Educação inclusiva e formação continuada: contribuições dos casos de ensino para os processos de aprendizagem e desenvolvimento profissional de 
professores. 2011. 351f. Tese (Doutorado em Educação) - Departamento de Educação, Universidade Federal do Rio Grande do Norte, Natal, 2011.

HAMMERNESS, K.; DARLING-HAMMOND, L.; SHULMAN, L. Toward Expert Thinking: how curriculum case-writing prompts the development of theory-based professional knowledge in student teachers. Teaching Education, Oxon, v.13, n. 2, p. 219-243, 2002.

MARCELO GARCIA, C. Formação de Professores: para uma mudança educativa. Porto: Porto Editora, 1999.

MERSETH, K. K. Cases and case methods in teacher education. In: SIKULA, J. (Ed.). Handbook of research on teacher education. New York: Macmillan, 1996. p.722744. Disponível em: <http://210.240.144.115/dyna/data/user/ndhu/files/201110141839070.pdf>. Acesso em: 30 mai. 2014.

MIZUKAMI, M. G. N. Aprendizagem da docência: algumas contribuições de L. S. Shulman. Revista Educação (UFSM), Santa Maria, RS, v.29, n.2, 2004. Disponível em: <http://coralx.ufsm.br/revce/index.htm>. Acesso em: 26 mai. 2014.

MIZUKAMI, M. G. N. et al. Escola e aprendizagem da docência: processos de investigação e formação. São Carlos: EdUFSCar, 2002.

MORAN, J. Os Modelos educacionais na aprendizagem on-line. [2011]. Disponível em: <http://www.eca.usp.br/prof/moran/site/textos/educacao_online/modelos.pdf >. Acesso em: 30 mai. 2014.

NONO, M. Caso de ensino e professores iniciantes. 2005. 238f. Tese (Doutorado em Educação) - Centro de Educação e Ciências Humanas, Universidade Federal de São Carlos, São Carlos, 2005.

Aprendendo a ensinar: futuras professoras das séries iniciais do ensino fundamental e casos de ensino. 2001. 176f. Dissertação (Mestrado em Educação) Centro de Educação e Ciências Humanas, Universidade Federal de São Carlos, São Carlos, 2001.

NÓVOA, A. (Coord.). Os Professores e sua Formação. Lisboa: Publicações Dom Quixote, 1992.

NÓVOA, A.; FINGER, M. (Org.). O Método (auto)biográfico e a formação. Natal: EDUFRN; São Paulo: Paulus, 2010.

PALLOFF, R. M.; PRATT, K. Construindo comunidades de aprendizagem no ciberespaço: estratégias eficientes para salas de aula on-line. Porto Alegre: ArtMed, 2002.

PIMENTA, S.; GHEDIN, E. (Org.). Professor reflexivo no Brasil: gênese e crítica de um conceito. 2.ed. São Paulo: Cortez, 2002. 
PORTAL dos professores [da UFSCar]. São Carlos. Apresenta a organização e recursos de portal dos professores. Disponível em: 〈http://www.portaldosprofessores.ufscar.br〉. Acesso: 26 mai. 2014.

SARMENTO, T. Contextos de Vida e Aprendizagem da Profissão. In: FORMOSINHO, J. (Coord.). Formação e Professores: aprendizagem profissional e acção docente. Porto: Porto Editora, 2009.

SCHÖN, D. A. Educando o profissional reflexivo: um novo design para o ensino e a aprendizagem. Porto Alegre: ArtMed, 2000.

SHULMAN, J. H. From practice to theory and back again: cases and portfolios as instruments for professional development. 2003. Disponível em:

<http://www.researchgate.net/publication/246254949_From_Practice_to_Theory_and_ Back_Again_Cases_and_Portfolios_as_Instruments_for_Professional_Developmenti>. Acesso em: 30 mai. 2014.

Happy accidents: cases as oportunities for teacher learning. In: ANNUAL MEETING OF THE AMERICAN EDUCATIONAL RESEARCH ASSOCIATION, 2002, New Orleans. Anais eletrônicos..., New Orleans, 2002. Disponível em: <http://cet.usc.edu/resources/teaching_learning/docs/happy_accidents.pdf $>$. Acesso em: 30 mai. 2014.

Case methods in teacher education. New York: Teaches College, 1992.

SHULMAN, L. S. Knowledge and Teaching: foundations of the new reform. Harvard Educational Review, Cambridge, v.57, n.1, p.1-22, 1987.

Those Who Understand: kwowledge growth in teaching. Educational

Researcher, Oxon, v.15, n.2, p.4-14, 1986. Disponível em:

<http://www.fisica.uniud.it/URDF/masterDidSciUD/materiali/pdf/Shulman_1986.pdf>. Acesso em: 30 mai. 2014.

SOARES, M. Alfabetização e Letramento. 2.ed. São Paulo: Contexto, 2004.

TANCREDI, R. M. S. P.; REALI, A. M. M. R; MIZUKAMI, M. G. N. Relatório do programa de mentoria para professores das séries iniciais. São Carlos, 2005.

Mimeografado.

WASSERMANN, S. Using cases to study teaching. Phi Delta Kappan, Bloomington, v.75, n.8, p.602-611, abr. 1994a.

Introduction to case method teaching: a guide to the galaxy. New York:

Teachers College, 1994b. 